\title{
Adaptive PI control and active tower damping compensation of a wind turbine
}

\author{
M. Lara, J. Garrido and F. Vázquez. \\ Computer Science and Numerical Analysis Department \\ University of Cordoba \\ Campus of Rabanales, Building Leonardo Da Vinci, 14071, Córdoba (Spain) \\ Phone/Faxnumber:+0034957218729; e-mail: p12laorm@uco.es,juan.garrido@uco.es and fvazquez@uco.es
}

\begin{abstract}
This paper deals with the control problem of a wind turbine model working in the nominal zone. This process is a nonlinear system whose dynamics vary strongly depending on the operation point. In the nominal region, the wind turbine speed is controlled by means of the pitch angle to generate the nominal power. The wind fluctuations and its non-uniform special profile act as disturbances on the power generation and the tower deflections. These oscillations must be reduced to improve the wind turbine lifetime.

In this work, an adaptive control structure operating on the pitch variable is proposed. It is composed of a gainscheduling PI control, an adaptive feedforward compensation of the wind speed and an adaptive gain compensation for the tower damping. The tuning of the controller parameters is formulated as an optimization problem that minimizes the tower fore-aft displacements and the deviation of the wind turbine speed from its nominal value. It is resolved using genetic algorithms for different linear models that are obtained from the nonlinear model.

The proposed controller is compared with a classical baseline PI (Proportional-integral) controller and the simulation results show a significant improvement of the system performance when the proposed strategy is applied.
\end{abstract}

\section{Key words}

Genetic algorithms (GA), Gain scheduling control, Wind turbine control, PI controller, Pitch angle control

\section{Introduction}

Due to global warming, there is currently a great interest in renewable energy, and in this context, wind energy has a leading role being one of the sources that has experienced the greatest growth in recent decades [1].

Taking into account that about $30 \%$ of greenhouse gas emissions are due to electricity generation, wind energy has had a great impulse as one of the main sources of renewable energy in order to reduce such emissions [2]. In fact, in the first decade of the millennium it maintained an average annual growth of $20 \%$ in the EU, $25 \%$ in the US and 50\% in China [3]. In 2018, with a net capacity of $189 \mathrm{GW}$ installed, it represents the second form of generating renewable energy in Europe [4].

It is necessary to reduce the costs of the total life cycle of the wind turbine, so that this energy source remains competitive against other alternative sources. This means reducing the initial investment, operation and maintenance costs [5]. These can be reduced by appropriate control strategies in combination with methods for monitoring the system health state [6].

\section{A. Operation regions}

Nowadays, horizontal axis variable speed and variable blade pitch (VS-VP) wind turbines with three blades are the most extended ones because they allow obtaining optimum power generation at different wind speeds [7]. $V S$-VP wind turbines can work in several operational modes or regions depending on the wind speed: cut-in (I); partial load (II); transition (III); full load (IV); and cut-out (V), as it is illustrated in Fig. 1, where the ideal power curve of wind turbine is shown:

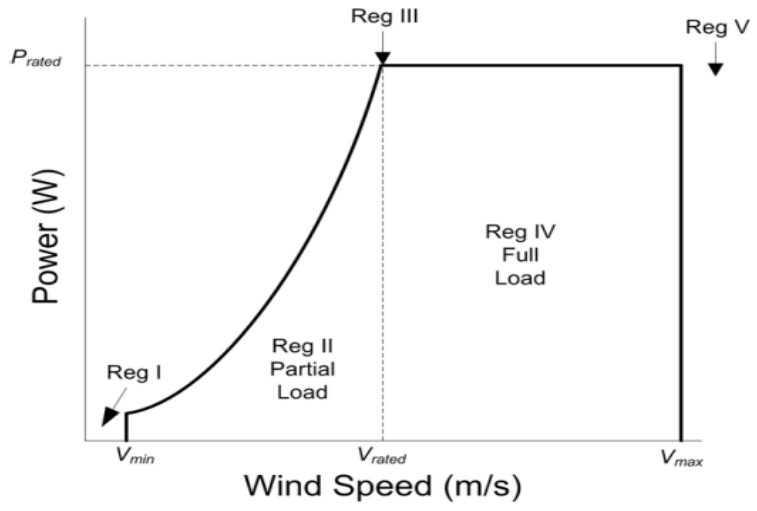

Fig.1. Operation regions of a wind turbine [3].

At the partial load region (region II), the wind turbine operates below the rated wind speed, trying to produce the maximum power. In this area, MPPT (Maximum Power Point Tracking) algorithms are used to obtain tracking set-points for output variables [8]. In this region, controllers act on the generator torque keeping the pitch angle constant in an optimum fixed value. In the region $I V$, full load region, the wind turbine has achieved 
the rated electric power and the controller must limit and smooth this power. In this case, the generator torque is kept constant and the pitch angle starts to operate to avoid the wind turbine overloading [9]. In the region III, a smooth transition between the previous two regions is carried out.

\section{B. Structural loads}

As wind turbines grow in size and power, the adverse effects of structural loads become more and more pronounced, especially those induced by aerodynamic and gravitational forces. Not mitigated structural loads can cause undesirable performance or even lead to early failure of the whole wind turbine system. Therefore, it is imperative to know how structural loads interact and influence in wind turbine power generation and how they affect its life time.

The most common phenomena that can cause different types of structural loads are the different deflection modes in the tower and rotor blades, or vibrations in the turbine drive train [10].

The tower structure mainly shows two vibration modes: fore-aft and side-to-side. The fore-aft movement is the tower rocking from back to front. There is a wide variety of works dealing with the mitigation of this mode [11], [12], [13].

Some commercial controllers use pitch control to actively damp the tower fore-aft ( $f$-a) motion when the wind turbine is operating at region IV. They usually add another control loop to the basic turbine speed PID controller and it is called Active Tower Damping Control (ATDC) [14]. For this control, the tower $f$-a acceleration is measured, integrated and multiplied by a gain. Then, this increment of pitch control is added to the baseline PI pitch control signal [15].

The paper is organized as follows: in section 2, some aspects of the wind turbine model are commented and a linearized model is presented for performing the control design. The proposed methodology of adaptive control structure is discussed in section 3. Section 4 shows a comparative analysis between the designed controller and a baseline controller. Finally, the conclusions are summarized in Section 5.

\section{Wind turbine model}

In this work, a wind turbine model is simulated using the Matlab/Simulink software with the assistance of FAST software (Fatigue, Aerodynamics, Structures and Turbulence) [16], [17]. The 5-MW NREL turbine used in this work has frequently been reported in the literature [17], which is based on the REpower 5 MW commercial model [18]. The 5-MW NREL turbine model has been implemented by using software FAST version 8 (v8.16.00a-bjj). Control loops and controllers are implemented in Matlab/Simulink.

\section{A. Linear models of the process}

The proposed design is based on linear models. Due to the nonlinearity of the system, their approximated linear models vary considerably depending on the operational point. In this work, an average linear model is obtained from the approximated linear models calculated at the seven operation conditions considered according to the disturbance value $\boldsymbol{v}_{\boldsymbol{w}}(12,14,16,18,20,22,24) \mathrm{m} / \mathrm{s}$ within the nominal region $(\boldsymbol{\omega} \boldsymbol{g}=1173.7 \mathrm{rpm})$. The linear models are computed using the Matlab Identification Toolbox.

The structure of the continuous model is given by equation (1), where $\boldsymbol{G}_{\boldsymbol{\omega}}(s)$ is the transfer function relating the angular velocity at high speed shaft $\left(\boldsymbol{\omega}_{\mathbf{g}}\right)$ to pitch angle $(\beta), \boldsymbol{G d}_{\boldsymbol{\omega}}(s)$ is the transfer function relating the controlled variable $\omega_{\mathbf{g}}$ to the wind disturbance variable $\left(\boldsymbol{v}_{\boldsymbol{w}}\right), \boldsymbol{G}_{\boldsymbol{x}_{\boldsymbol{t}}}(s)$ is the transfer function of the tower fore-aft displacement $\left(\mathbf{x}_{\mathbf{t}}\right)$ from the pitch $(\beta)$ and $\boldsymbol{G d}_{\mathbf{x}_{\mathbf{t}}}(s)$ is the transfer function relating the controlled variable $\mathbf{x}_{\mathbf{t}}$ to the wind disturbance variable $\left(\boldsymbol{v}_{w}\right)$.

$$
\begin{gathered}
G_{\omega g}(s)=\frac{K_{1}}{\left(s+p_{1}\right)} \\
G d_{\omega g}(s)=\frac{K_{2}}{\left(s+p_{2}\right)} \\
G_{\mathbf{x}_{\mathbf{t}}}(s)=\frac{K_{3}\left(s+z_{3}\right)}{\left(s+p_{31}\right)\left(s+p_{\left.32 a_{-}{ }^{+} p_{32 b} j\right)}\right.} \\
G d_{\mathbf{x}_{\mathbf{t}}}(s)=\frac{K_{4}\left(s+z_{4}\right)}{\left(s+p_{41}\right)\left(s+p_{\left.42 a_{-}{ }^{+} p_{42 b} j\right)}\right.}
\end{gathered}
$$

Although the model parameters of the different operation points are not shown, the obtained open loop dynamics of these models are all stable.

\section{Control methodology}

The control strategy proposed in this work combines two control loops: one loop to maintain the wind turbine speed at its nominal value and other loop to reduce the $f$-a tower displacements. The control scheme is depicted in Fig. 2. There is a gain-scheduling I-P controller regulating the turbine speed by actuating on the pitch angle. This loop also contains an adaptive feedforward compensation of the wind speed as a disturbance, that must be added to pitch control signal.

In addition, there is an adaptive proportional controller to mitigate the tower $f$-a oscillation that generates an extra pitch control component proportional to the tower fore-aft velocity $\dot{x}_{\boldsymbol{t}}$. This is also added to the pitch control signal. 


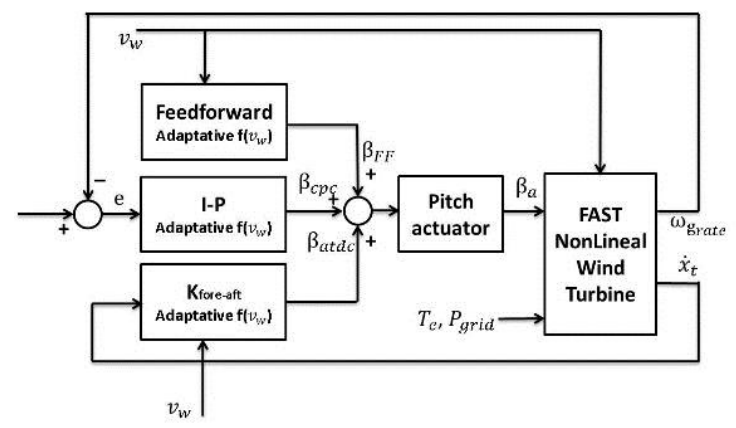

Fig.2. Pitch control system scheme

\section{A. Gain scheduling PI control}

Gain scheduling control consists in pre-setting a controller for various operating points, and subsequently, updating its parameters based on these designs and according to the operating point where the plant is working. There are different gain scheduling schemes in the literature. One of them is equivalent to several PI controllers working in parallel where only the output of one of them is chosen depending on the operating conditions. For instance, a very simple scheme of gain scheduling PI control is shown in Fig.3, where there are solely three previously designed controllers running in parallel and where the most appropriate one is selected based on the disturbance $d$. The other two not selected controllers are configured in integral tracking mode. Using a similar scheme in this work, an adaptive PI control is designed. It is composed of seven PI controllers tuned for the seven linear models obtained in the previous section. The value of the wind speed determines the PI controller that must be selected.

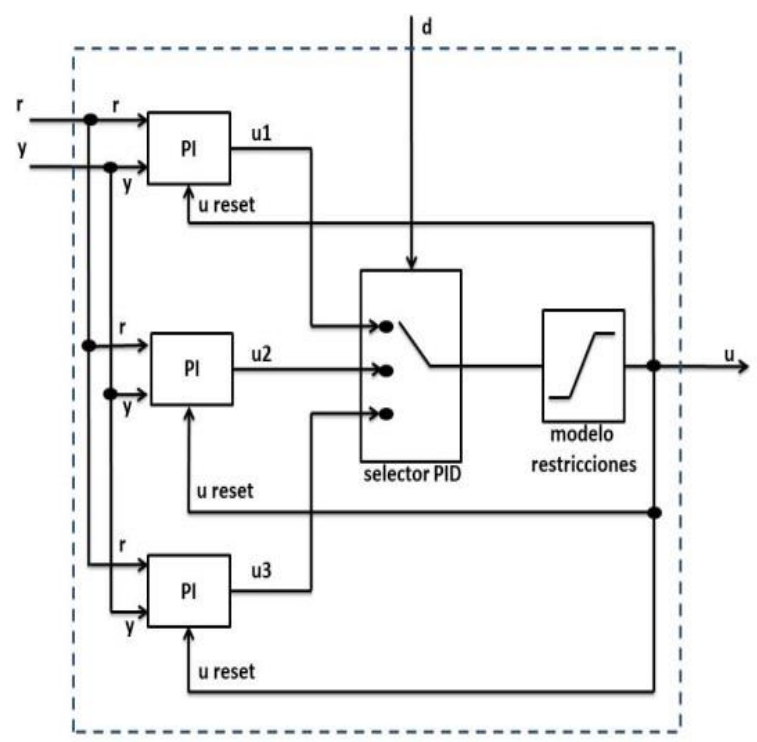

Fig.3. Adaptive PID Scheme

In order to assure a bumpless transfer between controllers without sudden changes in the control signal, all the PI controllers work in integral tracking mode updating their integral action so that the output of the unselected controllers matches with that of the active controller. It is similar to the mechanism used to achieve anti-windup and bumpless transfer between automatic/manual modes in PID controllers [19].

\section{B. Control I-P}

In this work the PI control is implemented with an I-P structure to avoid large control signals due to sudden set-point changes. The non-interactive I-P controller is composed by the following structure:

$$
u(s)=K_{p}\left(-y(s)+\frac{r(s)-y(s)}{T_{i} s}\right)
$$

where $u(s)$ is the control signal, $r(s)$ is the reference, $y(s)$ is the controlled signal, $K p$ is the proportional gain and Ti is the integral time constant. This continuous control law is discretized using the Tustin approximation. The proportional $P(k)$ and integral $I(k)$ actions of this implementation in the $k$-th iteration are detailed below:

$$
\begin{gathered}
P(k)=K_{p}(-y(s)) \\
I(k)=I(k-1)+K_{p i}(e(k)+e(k-1))
\end{gathered}
$$

The constant $K_{p i}$ is given by:

$$
K_{p i}=K_{p} \frac{h}{2 \cdot T_{i}}
$$

where $h$ is the sample time. The control signal is the sum of these two actions and, additionally, a feedforward action $F F(k)$ and a fore-aft action $F A(k)$. Therefore, the control signal is given by $u(k)=P(k)+I(k)+F F(k)+F A(k)$.

To cope with the input constraints of the process, an antiwindup mechanism is implemented using an input constraint model that considers the input saturations. When the final control signal $u(k)$ is out of its limits, this mechanism updates the integral term $I(k)$ constrained $u(k)$ to the exceeded limit.

The mechanism is shown in (5). In addition, to ensure the tracking of the rest of the controllers to the final control signal $u(k)$, it must be considered that the variables $P$ $(k), I(k)$ and $U(k)$ are vector signals of seven elements, one for each controller of the adaptive control. As a result, the signal $I(k)$ is updated in the else statement in such a way the rest of the non-active controllers follow the final signal $u(k)$

$$
\begin{aligned}
& u(k)=P(k)+I(k)+F F(k)+F A(k) \\
& \text { if } u(k)>u_{\text {max }} \\
& \quad I(k)=u_{\text {max }}-P(k)-F F(k)-F A(k) \\
& \text { elseif } u(k)<u_{\text {min }} \\
& \quad I(k)=u_{\min }-P(k)-F F(k)-F A(k) \\
& \text { else } \quad I(k)=u(k)-P(k)-F F(k)-F A(k) \\
& \text { end }
\end{aligned}
$$

\section{Adaptive feedforward}

The feedforward control strategies have already been used in literature to control wind turbines [20, 21]. They are usually combined with a PID control so that the advantages of each one are added. The joint of both control signals can significantly improve the performance of the system in those cases in which the disturbance can be measured before it affects the process output. 
Adaptive feedforward control is performed based on the seven linear models identified previously and calculated according to (1). Depending on wind speed $v_{w}$, the value of the gain and time constant of the dynamics $G_{\omega g}$ and $G d_{\omega g}$ are modified linearly and the transfer function of the feedforward compensation $\mathrm{Ca}(\mathrm{s})$ is updated according to (6).

$$
C a(s)=\frac{-G d_{\omega g}(s)}{G_{\omega g}(s)}=\frac{-\frac{K_{v i}}{T p_{v i} s+1}}{\frac{K_{\text {pitch }}}{T p_{\text {pitch }} s+1}}
$$

\section{E. Controller tuning by genetic algorithms}

The tuning procedure of the PI controllers with fore-aft control is performed by means of a multi-objective genetic algorithm (MGA) simulating each of the seven linear models. The function to minimize is composed by the following indices in (7): integral of time-weighted absolute error (ITAE) and integral of absolute error (IAE) of the controlled variable $\omega_{\mathrm{g}}$, and the cumulative variation rate $(C V R)$ and ITAE of the variable $\mathrm{x}_{\mathrm{t}}$ taking as reference the stationary value for this last index.

$$
\begin{aligned}
I A E_{\omega g} & =\int_{t_{0}}^{t_{s}}\left|\omega_{g_{\text {rat }}}-\omega_{g}(t)\right| d t \\
I T A E_{\omega g} & =\int_{t_{0}}^{t_{s}} t\left|\omega_{g_{-} r a t}-\omega_{g}(t)\right| d t \\
C V R_{x_{\boldsymbol{t}}} & =\int_{0}^{t_{s}}\left|\boldsymbol{x}_{\boldsymbol{t}}\left(t_{k}\right)-\boldsymbol{x}_{\boldsymbol{t}}\left(t_{k-1}\right)\right| d t \\
I T A E_{\boldsymbol{x}_{\boldsymbol{t}}} & =\int_{0}^{t s} t\left|\boldsymbol{x}_{\boldsymbol{t}}(t)-\boldsymbol{x}_{\boldsymbol{t}}(\infty)\right| d t
\end{aligned}
$$

Due to the nonlinearity of these performance indices, the PI tuning design that minimizes them must be formulated as a nonlinear optimization problem, and thus, it can be solved using MGA [22], [23]. The procedure is performed using the Global Optimization toolbox of Matlab. In each PI controller, there are three parameters to be tuned: Kp and Ti (PI controller) and $K_{\text {fore_aft }}(A T D C)$. The parameter search range is limited from $10^{-4}$ to 30 . For the Kp gain, this range is negative. The main options configured in the genetic algorithm are: a population size of 5000, elite count of 0.05 times the population size for reproduction with a crossover fraction of 0.8 .

The simulation is optimized for each linear model obtained as follows: a multi-objective optimization is carried out considering the previously mentioned indices (7), where the controller must maintain the nominal angular velocity $\boldsymbol{\omega}_{\mathbf{g}}=1173.7 \mathrm{rpm}$ for wind changes of $\pm 0.5 \mathrm{~m} / \mathrm{s}$ in each operating point. The linear model also takes into account the noise model presented by the nonlinear model in the variable $\omega_{\mathrm{g}} y \mathrm{x}_{\mathrm{t}}$, obtaining a better fit. The designed cost function seeks to reduce the fatigue suffered by the tower through minimizing fore-aft displacements at the same time that try to maintain the turbine speed close to the nominal value.

\section{Simulation results}

In this section, the performance of the proposed controller is evaluated: the designed controller is compared to a traditional gain scheduling PI controller. The most common structure for this baseline controller is represented in Fig. 4. Proportional and integral parameters are defined as $\boldsymbol{K}_{\boldsymbol{p}}=0.0188 \cdot f(\beta)$ and $\boldsymbol{K}_{\boldsymbol{i}}=0.00807 \cdot f(\beta)$, respectively, and $f(\beta)=1 /(1+\beta / 0.11)$, is the programming function of the PI controller [17].

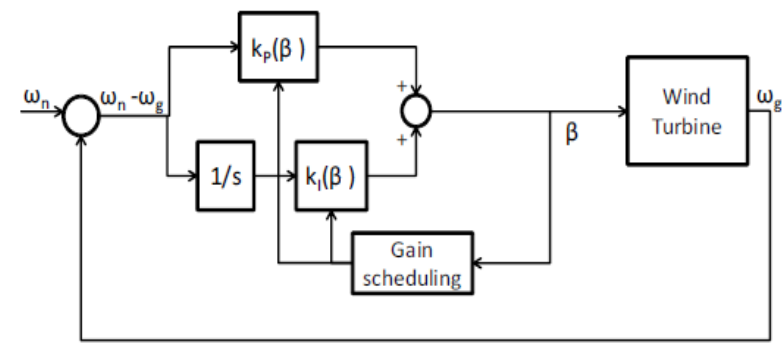

Fig.4. The baseline controller structure [24]

The $5 \mathrm{MW}$ wind turbine is directly operated in FAST in Region IV, where the rated generator speed $\omega_{g}$ is 1173.7 rpm. In order to obtain simulation data that can be compared, the wind turbine was operated with constant wind speed. In different simulations, the wind speed is set to have several steps changes such as 12 to 14,14 to 16 , 16 to 18,18 to 20,20 to 22 and 22 to $24 \mathrm{~m} / \mathrm{s}$, respectively. Thus, it is possible to compare the control performance of both control systems for each operation point.

The step responses are shown in Fig.5. The first plot shows the generator speed, the second one, the tower top fore-aft displacement and the last one, the pitch angle.

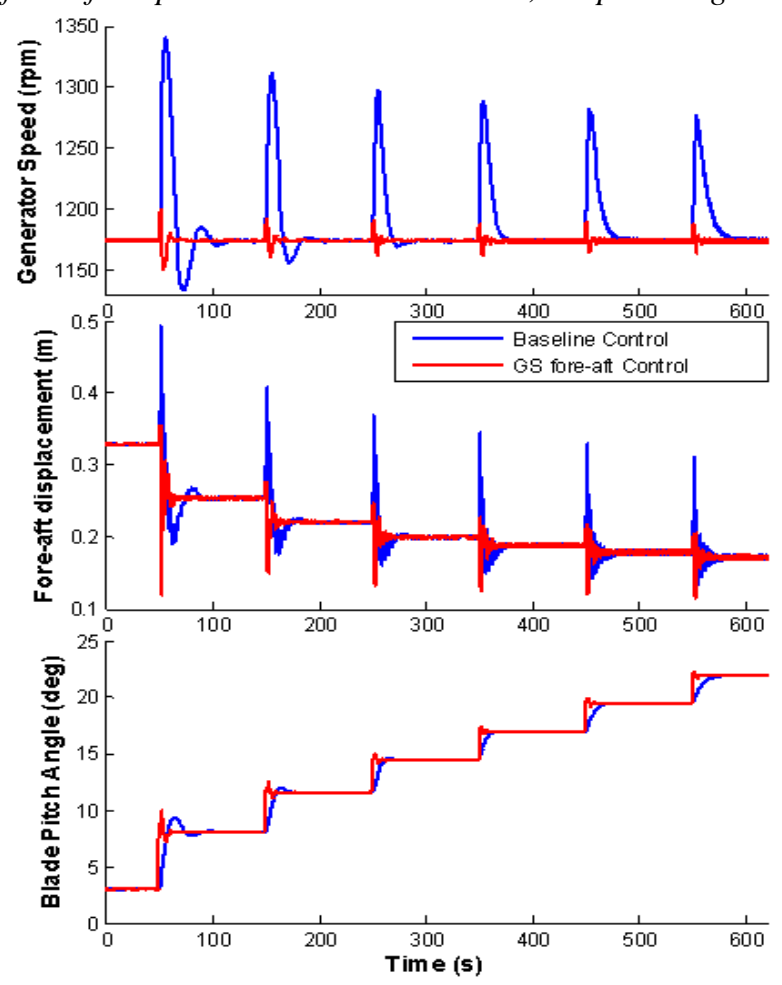

Fig.5. Comparative control system responses to wind steps 
The simulation results of this figure show that the designed controller works better than the baseline controller for all wind speeds. In order to perform a comparative quantitative analysis, the performance indices are calculated from the simulation data. The corresponding results are shown in Table 1.

Table 1. Performance index values for steps wind speeds (Baseline Controller BC and Gain Scheduled Controller GSC)

\begin{tabular}{|c|c|c|c|c|c|c|}
\hline \multicolumn{2}{|c|}{$\begin{array}{c}\text { Wind speed } \\
\text { steps/Indices }\end{array}$} & IAE $_{\boldsymbol{\omega g}}$ & ITAE $_{\boldsymbol{\omega g}}$ & CVR $_{\mathbf{x}_{\mathbf{t}}}$ & ITAE $_{\mathbf{x}_{\mathbf{t}}}$ & $\mathbf{C V R}_{\boldsymbol{\beta}}$ \\
\hline \multirow{2}{*}{ Total } & $\mathbf{B C}$ & $8.7 e 3$ & $10.1 e 4$ & 7.11 & $2.55 e 3$ & 25.6 \\
\cline { 2 - 7 } & $\mathbf{G S C}$ & 754.2 & $19.2 e 3$ & 4.83 & $1.07 e 3$ & 60.5 \\
\hline $\mathbf{1 2 - 1 4}$ & $\mathbf{B C}$ & $2.2 e 3$ & $2.98 e 4$ & 1.07 & 769.11 & 8.6 \\
\cline { 2 - 7 }$(\mathbf{m} / \mathbf{s})$ & $\mathbf{G S C}$ & 181.7 & $2.35 e 3$ & 0.85 & 118.68 & 16.0 \\
\hline $\mathbf{1 4 - 1 6}$ & $\mathbf{B C}$ & $1.5 e 3$ & $1.58 e 4$ & 1.06 & 317.95 & 4.8 \\
\cline { 2 - 7 }$(\mathbf{m} / \mathbf{s})$ & $\mathbf{G S C}$ & 114.1 & $2.21 e 3$ & 0.65 & 83.89 & 10.6 \\
\hline $\mathbf{1 6 - 1 8}$ & $\mathbf{B C}$ & $1.2 e 3$ & $1.17 e 4$ & 1.11 & 294.14 & 3.4 \\
\cline { 2 - 7 }$(\mathbf{m} / \mathbf{s})$ & $\mathbf{G S C}$ & 98.5 & $2.54 e 3$ & 0.72 & 128.79 & 8.6 \\
\hline $\mathbf{1 8 - 2 0}$ & $\mathbf{B C}$ & $1.1 e 3$ & $1.16 e 4$ & 1.15 & 395.67 & 2.9 \\
\cline { 2 - 7 }$(\mathbf{m} / \mathbf{s})$ & $\mathbf{G S C}$ & 103.2 & $3.13 e 3$ & 0.73 & 164.49 & 7.1 \\
\hline $\mathbf{2 0 - 2 2}$ & $\mathbf{B C}$ & $1.2 e 3$ & $1.45 e 4$ & 1.25 & 404.78 & 2.8 \\
\cline { 2 - 7 }$(\mathbf{m} / \mathbf{s})$ & $\mathbf{G S C}$ & 120.3 & $4.36 e 3$ & 0.81 & 276.47 & 8.6 \\
\hline $\mathbf{2 2 - 2 4}$ & $\mathbf{B C}$ & $1.3 e 3$ & $1.72 e 4$ & 1.34 & 372.39 & 2.7 \\
\cline { 2 - 7 }$(\mathbf{m} / \mathbf{s})$ & $\mathbf{G S C}$ & 121.2 & $4.69 e 3$ & 0.85 & 302.15 & 8.3 \\
\hline
\end{tabular}

All index values related to errors have been improved at the expense of a greater control effort $\boldsymbol{C V}_{\boldsymbol{\beta}}$ in the manipulated variable $(\beta)$. It is important to clarify that the variations of step changes in wind speed are really unrealistic. However, this is a very strong requirement for the control system, which must overcome the rapid and abrupt change. In Fig.6, one of the responses to a wind step change is zoomed to get a better appreciation of the improvement of the proposed design: there is a faster disturbance rejection of the wind speed improving the tracking of $\mathbf{\omega}_{\mathbf{g}}$ and fluctuations in the controlled variable $\mathbf{x}_{\mathbf{t}}$ are smaller.

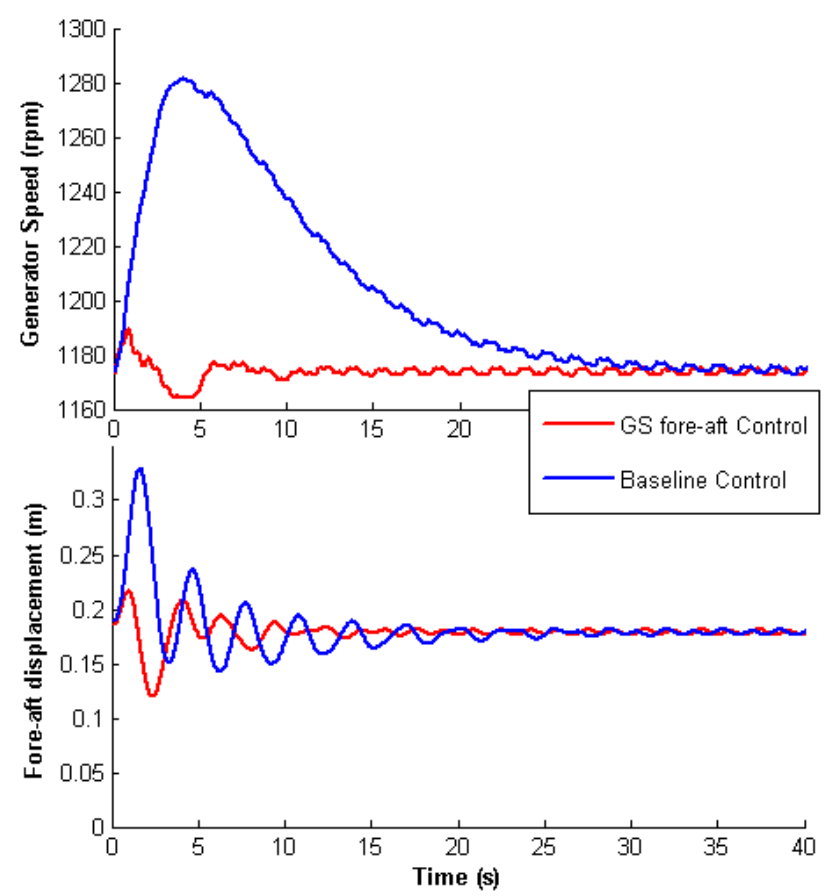

Fig.6. Generator speed and fore-aft displacement to a wind speed step change from 20 to $22 \mathrm{~m} / \mathrm{s}$
Next to perform a simulation with a more realistic wind speed profile, a stochastic wind speed has been used in the model. This wind has the following characteristics: a mean value of $17 \mathrm{~m} / \mathrm{s}$, a turbulent component with standard deviation of $10 \%$ and a sine component with amplitude of $1 \mathrm{~m} / \mathrm{s}$ and period of $100 \mathrm{~s}$.

Fig.7 shows the simulation results using this wind speed profile. The first plot shows the wind speed, the second the generator speed, the third one the tower top fore-aft displacement and last one the pitch angle.
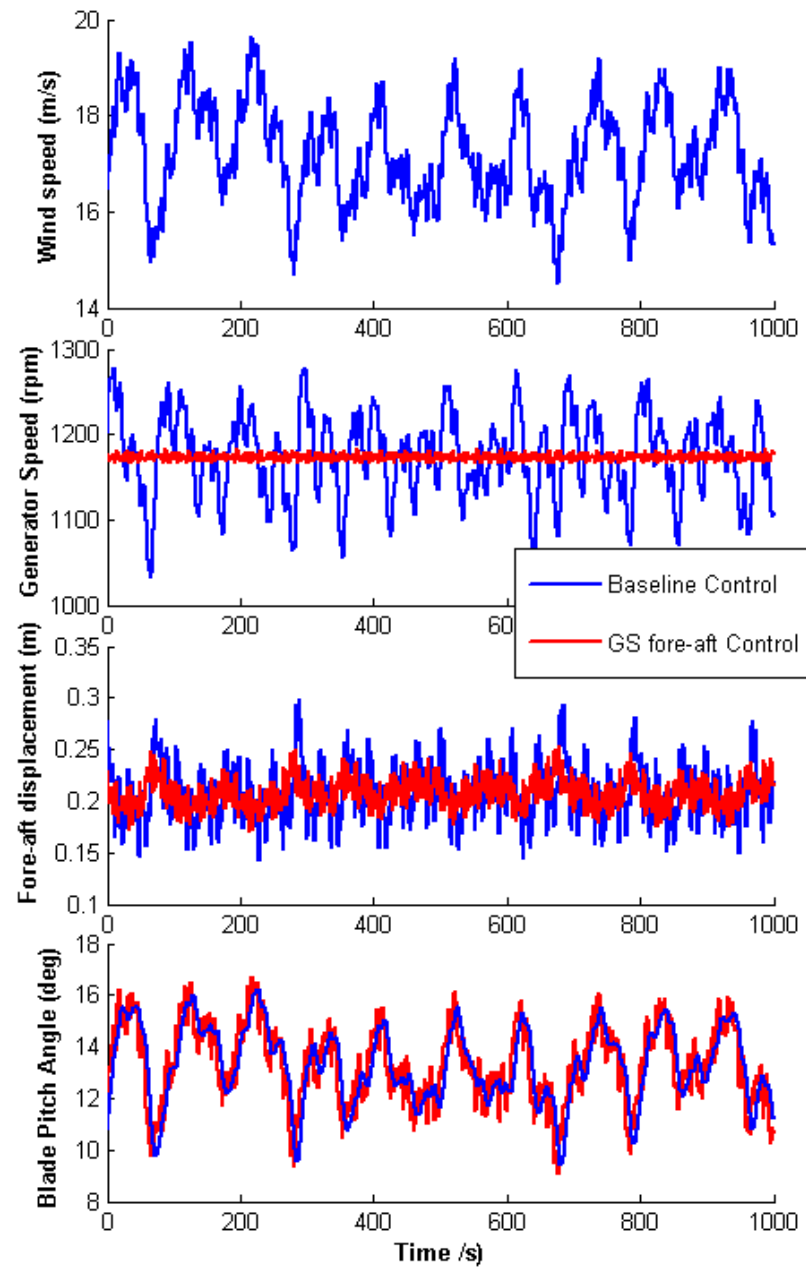

Fig.7. Simulation responses with a stochastic wind speed profile

The corresponding performance indices are collected in Table 2.

Table 2. Performance index values for stochastic wind speeds (Baseline Controller BC and Gain Scheduled Controller GSC)

\begin{tabular}{|c|c|c|c|}
\hline & IAE $_{\boldsymbol{\omega g}}$ & $\mathbf{C V R}_{\mathbf{x}_{\mathbf{t}}}$ & $\mathbf{C V R}_{\boldsymbol{\beta}}$ \\
\hline $\mathbf{B C}$ & $4.08 e+04$ & 13.14 & 118.76 \\
\hline $\mathbf{G S C}$ & $3.18 e+03$ & 8.89 & 341.09 \\
\hline
\end{tabular}

Observing the qualitative and quantitative results, the following can be highlighted: the proposed controller obtains a better $C V R_{x_{t}}$ index value, reducing its index in a $32.3 \%$ compared to that of the baseline controller. With respect to the $I A E_{\omega g}$ value, the designed controller reduces this index in a $92.2 \%$ in comparison with that obtained using the baseline controller. 
Regarding the qualitative and quantitative results against step wind, similar conclusions can be obtained: the designed controller obtains a better $C V R_{x_{t}}$ and ITAE $E_{x_{t}}$ index values, reducing such index values in a $32.06 \%$ and $58.04 \%$, respectively, in comparison with those of the baseline controller. With respect to the $I A E_{\omega g}$ and ITAE $E_{\omega g}$ index values, the designed controller reduces these indices in a $91.3 \%$ and $81 \%$ respectively, compared to the baseline controller.

\section{Conclusions}

An adaptive wind turbine control structure operating on the pitch variable in the nominal zone is developed in this work. The proposed control strategy mainly combines two control loops: the first one to maintain the wind turbine speed in its nominal value and the second to reduce the f-a tower displacements. The gain-scheduling I$P$ controller with adaptive feedforward regulates the turbine speed by actuating on the pitch angle, and the active tower damping control generates an extra pitch control component proportional to the tower fore-aft velocity reducing the structural fatigue of the tower. The tuning procedure of the PI controllers with fore-aft control is performed by means of a multi-objective genetic algorithm seeking to minimize the tower fore-aft displacements and the deviation of the wind turbine speed from its nominal value.

The proposed controller is compared with a classic adaptive controller using different wind conditions. The simulation results show a significant improvement in the performance of the designed controller for both step and stochastic winds, improving the reference tracking of the turbine angular speed and mitigating the tower $f$-a oscillations in comparison with the baseline controller.

\section{Acknowledgement}

M. Lara would like to express appreciation for the FPU fellowship (FPU17/02747) from the Spanish Ministry of Education, Culture, and Sports

\section{References}

[1] Njiri, J. G., \& Söffker, D. (2016). State-of-the-art in wind turbine control: Trends and challenges. Renewable and Sustainable Energy Reviews, 60, 377-393.

[2] Kaldellis, J. K., \& Zafirakis, D. (2011). The wind energy revolution: A short review of a long history. Renewable Energy, 36(7), 1887-1901.

[3] Fragoso, S., Garrido, J., Vázquez, F., \& Morilla, F. (2017). Comparative analysis of decoupling control methodologies and $H \infty$ multivariable robust control for variable-speed, variable-pitch wind turbines: Application to a lab-scale wind turbine. Sustainability (Switzerland), 9(5).

[4] Walsh, C., \& Pineda, I. (2019). Wind energy in Europe in 2018. 32.

[5] Myhr, A., Bjerkseter, C., Ågotnes, A., \& Nygaard, T. A. (2014). Levelised cost of energy for offshore floating wind turbines in a lifecycle perspective. Renewable Energy, 66, 714-728.

[6] Tchakoua, P., Wamkeue, R., Ouhrouche, M., SlaouiHasnaoui, F., Tameghe, T. A., \& Ekemb, G. (2014). Wind turbine condition monitoring: State-of-the-art review, new trends, and future challenges. Energies, 7(4), 2595-2630.

[7] Hansen, A. D. (2005). Generators and power electronics for wind turbines. Wind power in power systems (pp. 53 78).

[8] Kumar, D., \& Chatterjee, K. (2016). A review of conventional and advanced MPPT algorithms for wind energy systems. Renewable and Sustainable Energy Reviews, 55, 957-970.

[9] Boukhezzar, B., Lupu, L., Siguerdidjane, H., \& Hand, M. (2007). Multivariable control strategy for variable speed, variable pitch wind turbines. Renewable Energy, 32(8), 1273-1287.

[10] Njiri, J. G., \& Söffker, D. (2016). State-of-the-art in wind turbine control: Trends and challenges. Renewable and Sustainable Energy Reviews, 60, 377-393.

[11] Liu, H., Tang, Q., Chi, Y., Zhang, Z., \& Yuan, X. (2016). Vibration reduction strategy for wind turbine based on individual pitch control and torque damping control. International Transactions on Electrical Energy Systems, 26(10), 2230-2243.

[12] Mohammadi, E., Fadaeinedjad, R., \& Moschopoulos, G. (2018). Implementation of internal model based control and individual pitch control to reduce fatigue loads and tower vibrations in wind turbines. Journal of Sound and Vibration, 421, 132-152.

[13] Gambier, A., \& Nazaruddin, Y. Y. (2018). Collective pitch control with active tower damping of a wind turbine by using a nonlinear PID approach. IFAC-PapersOnLine, 51(4), 238-243.

[14] Bossanyi, E., 2000. The design of closed loop controllers for wind turbines. Wind Energy, pp. 149-163.

[15] Bossanyi, E. A., 2003. Wind turbine control for load reduction. Wind Energy, p. 229-244.

[16] Jonkman, J., Jonkman, B. NWTC Information Portal (FAST v8). https://nwtc.nrel.gov/FAST8 (Last visit 1-10-2019).

[17] J. M. Jonkman, S. Butterfield, W. Musial, G. Scott, Definition of a 5-MW reference wind turbine for offshore system development, Tech. rep., National Renewable Energy Laboratory, Golden, Colorado, nREL/TP-50038060 (2009).

[18] REpower. http://www.repoweringsolutions.com/ (Last visit 1-10-2019).

[19] Garrido, J., Lara, M., Ruz, M., Vázquez, F., Alfaya, J. A., Morilla, F. (2018). Decentralized PID control with inverted decoupling and superheating reference generation for efficient operation: Application to the Benchmark PID 2018. 3rd IFAC Conference on Advances in PID Control. Ghent, Belgium, pp. 710-715.

[20] Ungurán, R., Petrović, V., Pao, L. Y., \& Kühn, M. (2019). Uncertainties identification of the blade mounted lidarbased inflow wind speed measurements for robust feedback-feedforward control synthesis. Wind Energy Science Discussions, (April), 1-29.

[21] Chengyuan Yu, Dongdong Li "Fuzzy-PI and feedforward control strategy of DFIG wind turbine," 2012 IEEE Innovative Smart Grid Technologies - Asia, ISGT Asia 2012, art. no. 6303299.

[22] Goldberg, D.E. (1989). Genetic Algorithms in Search, Optimization \& Machine Learning, Addison-Wesley.

[23] Wang, P., Kwok, D.P. (1994). Optimal design of PID process controllers based on genetic algorithms, Control Eng. Pract., 2(4), 641-648.

[24] O. Bagherieh and R. Nagamune, "Gain-scheduling control of a floating offshore wind turbine above rated wind speed," Control Theory Technol., vol. 13, no. 2, pp. 160 172, 2015. 